\title{
The Cross-Calibration of Fermi-GBM and INTEGRAL-ISGRI by Spectral Analysis of a Sample of Simultaneously Observed GRBs
}

\author{
Dave Tierney $^{a}$ and Sheila McBreen ${ }^{a b}$ on behalf of the Fermi GBM Team, Lorraine \\ Hanlon $^{a}$ Suzanne Foley ${ }^{a b}$ Antonio Martin-Carrillo ${ }^{a}$ Martin Topinka ${ }^{a}$ Seamus \\ Meehan $^{a}$ \\ ${ }^{a}$ University College, Dublin, Belfield, Stillorgan Road, Dublin 4, Ireland \\ ${ }^{b}$ Max-Planck-Institut fur extraterrestrische Physik (Giessenbachstrasse 1, 85748 Garching, \\ Germany)
}

E-mail: david.tierneyducd.ie

We present results of a cross-calibration study between the Fermi Gamma-Ray Burst Monitor (GBM) and the INTEGRAL Soft Gamma-Ray Imager ISGRI. For the study, the spectral analysis of a sample of 4 Gamma-Ray Bursts (GRBs) simultaneously observed by both instruments were taken into consideration. Spectral models were fit separately for GRB081226B, GRB090107B, GRB090625B and GRB090704 using data sets from both instruments. A joint spectral model was then fit to the data to calculate the normalisation factor between the spectral models. With the overlap in lifetimes of the Fermi and INTEGRAL satellites and given that the detection rate for a common burst is $\sim 1$ every 4 months, more bursts will be added to the sample in future years

8th INTEGRAL Workshop "The Restless Gamma-ray Universe"- INTEGRAL 2010,

September 27-30, 2010

Dublin Ireland 


\section{Objectives}

The INTErnational Gamma-Ray Astrophysics Laboratory (INTEGRAL) has been in operation since 2002 [四] and the imager ISGRI [] is well calibrated [B]]. Fermi Gamma-Ray Burst Monitor (GBM) [四] has been in operation since 2008 and has detected over 500 GRBs to date. After

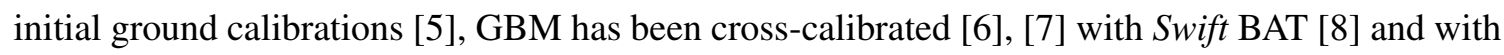
INTEGRAL SPI [ [9] by means of simultaneously observed GRBs.

The cross-calibrations of Fermi with other satellites during the mission phase are necessary to either i) ensure consistency between the current calibration and ground calibration or ii) apply a modification to the pre-launch ground calibration efforts. The change in environment, temperature, pressure and the stress of the launch can alter the ground calibration of the satellite. The GBM detector response can potentially be modified if necessary by comparing GRB spectral parameters with a well calibrated instrument.

The objective of this study was to carry out a cross calibration between Fermi GBM and INTEGRAL ISGRI by analysing bursts common to both instruments. ISGRI has been in operation from 2002 and has been calibrated numerous times in its lifetime. Over the energy range $8 \mathrm{keV}$ $1 \mathrm{MeV}$ ISGRI can detect $\sim 10$ times lower peak flux $\left(\mathrm{ph} \mathrm{s}^{-1} \mathrm{~cm}^{-2}\right)$ than GBM, thus ISGRI has a greater spectral sensitivity over this range compared with GBM.

To compare the 2 instruments, the spectral parameters are fit separately for each instrument, to determine differences in spectral parameters over the individual energy ranges of the instruments. By fitting the two detectors in a joint fit, the difference in flux measured by each instrument could be measured via a calibration constant.

\section{Instrumentation}

The Fermi Gamma-ray Space Telescope is an astronomical satellite for observing the gammaray sky, with one of the objectives to observe and study gamma-ray transients, specifically GammaRay Bursts (GRBs). It has an energy range spanning several decades $(\sim 8 \mathrm{keV}-\sim 300 \mathrm{GeV})$ using 2 instruments - the Large Area Telescope (LAT) [ए]] operating between $\sim 20 \mathrm{MeV}-\sim 300 \mathrm{GeV}$ and the Gamma-Ray Burst Monitor (GBM) operating between $8 \mathrm{keV}-30 \mathrm{MeV}$.

GBM further consists of two types of detectors - 12 Sodium Iodine (NaI) scintillating crystals operating between $8-1000 \mathrm{keV}$ and $2 \mathrm{BGO}$ scintillating crystals operating between $0.15-30 \mathrm{MeV}$. The field of view of GBM is the entire unocculted (by the Earth) sky, $\sim 8$ steradians.

INTEGRAL was designed for a nominal lifetime of 5 years and in 2009 it was decided to extend the mission at least up to the end of 2012. The INTEGRAL satellite contains two gammaray observing instruments - a spectrometer SPI and an imager IBIS. For the purpose of this crosscalibration the ISGRI detector on IBIS was used as it operates in the energy range $8 \mathrm{keV}-1 \mathrm{MeV}$.

The ISGRI dectector operates by use of a coded mask comprising of a tungsten shield and CdTe detection pixels. ISGRI has a fully coded field of view of $9^{\circ} \times 9^{\circ}$ with an angular resolution of 12'. The effective area of ISGRI falls off rapidly $\sim$ before $20 \mathrm{keV}$ and after $200 \mathrm{keV}$. ISGRI is spectrally, more sensitive than GBM but as its effective area drops rapidly outside this range meaning it is only efficient in the $20-200 \mathrm{keV}$ range.

From July 2008 to November 2010 GBM has detected nearly 600 GRBs at a rate of $\sim 21$ per month with ISGRI detecting 25 in this time frame at a rate of just under $1 /$ month. There have been 
8 bursts common to both instruments between July 2008 to November 2010 and they represent the complete set of Fermi GBM and INTEGRAL ISGRI GRBs. From analysing the data this leads to a prediction of $\sim 1$ GRB every 4 months detected by both instruments.

Table 1 shows the four bursts that were studied in detail for this cross calibration effort

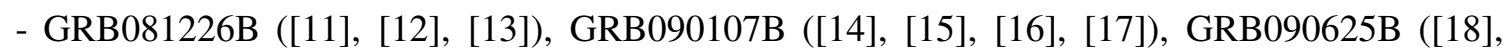
[एव] , [20]) and GRB090704 ([2]], [2]). Out of the 4 bursts studied, 3 are long and one burst, GRB081226B, is short.This can be seen in Figure 1 in which the light curves measured by ISGRI and GBM can be compared. The location of each burst was taken from the best ISGRI / XRT localisation.

\begin{tabular}{|l|l|l|l|l|}
\hline \multicolumn{5}{|c|}{ Common GRBs } \\
\hline GBM Name & Standard Name & $T_{0}$ INTEGRAL & $T_{0}$ Fermi & Temporal Selections \\
\hline GRB081226.509 & GRB081226B & $12: 13: 11$ & $12: 13: 10.71$ & $12: 13: 10.61-12: 13: 10.91$ \\
\hline GRB090107.681 & GRB090107B & $16: 20: 36$ & $16: 20: 42.77$ & $16: 20: 38-16: 20: 54$ \\
\hline GRB090625.560 & GRB090625B & $13: 26: 20$ & $13: 26: 22.51$ & $13: 26: 20-13: 26: 28$ \\
\hline GRB090704.242 & GRB090704 & $05: 47: 43$ & $05: 47: 48.18$ & $05: 47: 48-05: 48: 51$ \\
\hline
\end{tabular}

\section{Comparison of Data}

The software used to analyse the data were: Off-line Scientific Analysis (OSA) $5 \& 7$ for reduction of ISGRI data (OSA 5 was used to analyse the short burst GRB081226B), RMFIT [[23] for reduction of GBM data and XSPEC [24] for spectral analysis and final comparison. To ensure that different data sets were comparable and for the accuracy of the cross calibration four factors were taken into account when comparing the data - temporal binning, time intervals, energy ranges and energy binning.

As GBM is comprised of 14 separate detectors, the selection of detectors is important for the analysis of the data. A tool was used to detect blockages between the GRB line of sight and individual detectors to ensure that absorption of gamma-rays passing through the satellite were accounted for in the analysis. As a general rule, detectors were selected that had a source angle of $<60^{\circ}$.

Once the data were prepared it were analysed in XSPEC11. Two models were selected for this analysis, a simple power law (PL) and a high energy exponential cut off PL (COPL). A Band model [25] was also used to fit the joint data sets as a check if there was any impovement using this model over a COPL. There was no significant improvement in the statistics to justify the addition of an extra model parameter. The goodness of fit of the spectral models of the data was determined using $\chi^{2}$ statistics.

The PL and COPL model were multiplied by a normalisation constant. The similar parameters for each data set were tied with the exception of the constant. By doing this the ratio of the normalisation factors can be calculated. For the GBM data this was set to 1 and for the ISGRI data set this was allowed to vary. Using this method, both data sets were fit with the same model with the same parameters in each apart from the constant. 
(a)

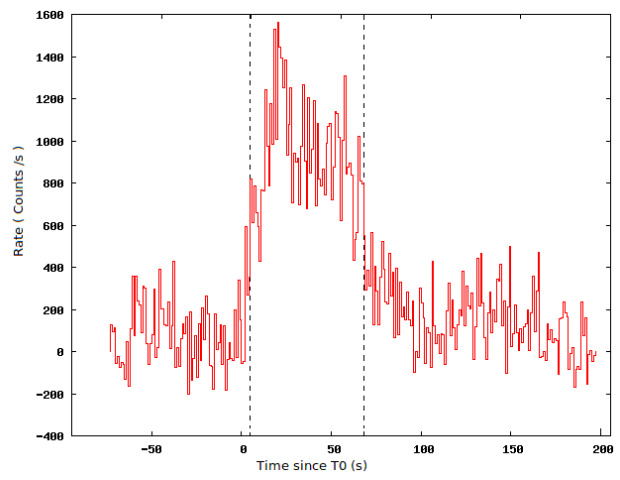

(c)

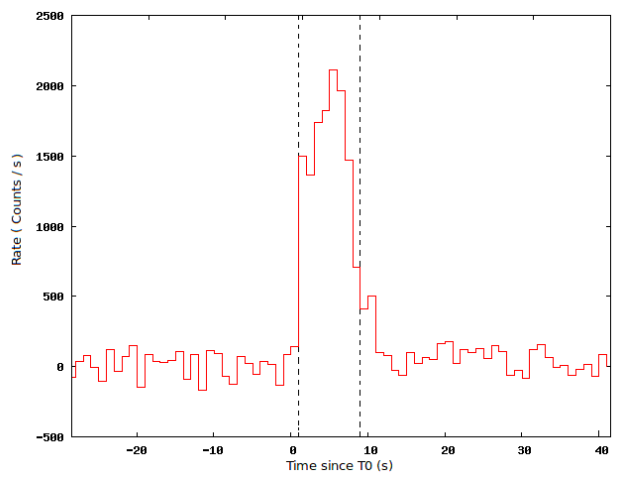

(d)

(e)

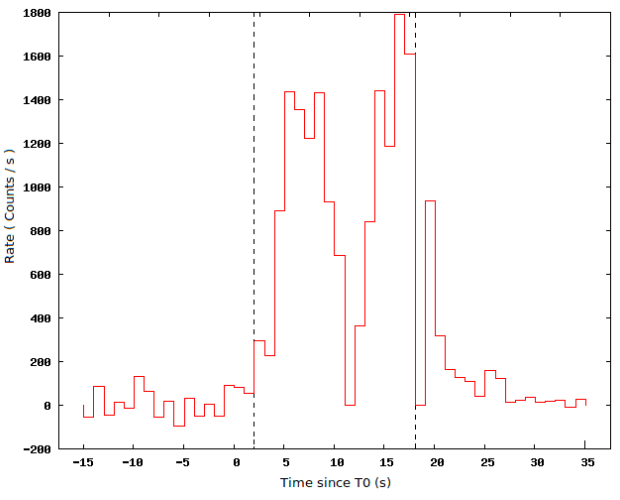

(g)

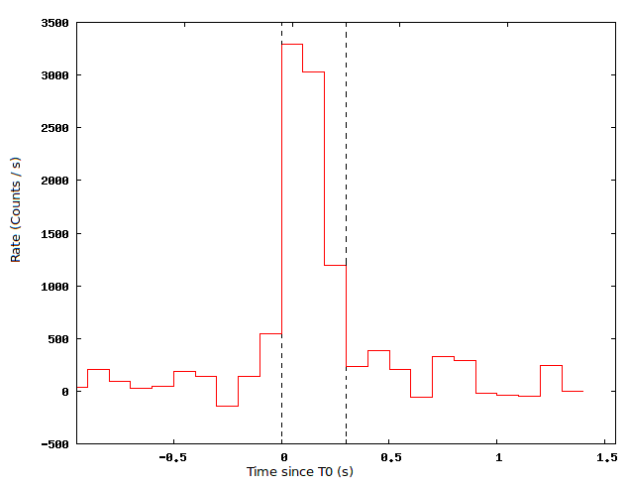

(h)

(b)

(f)
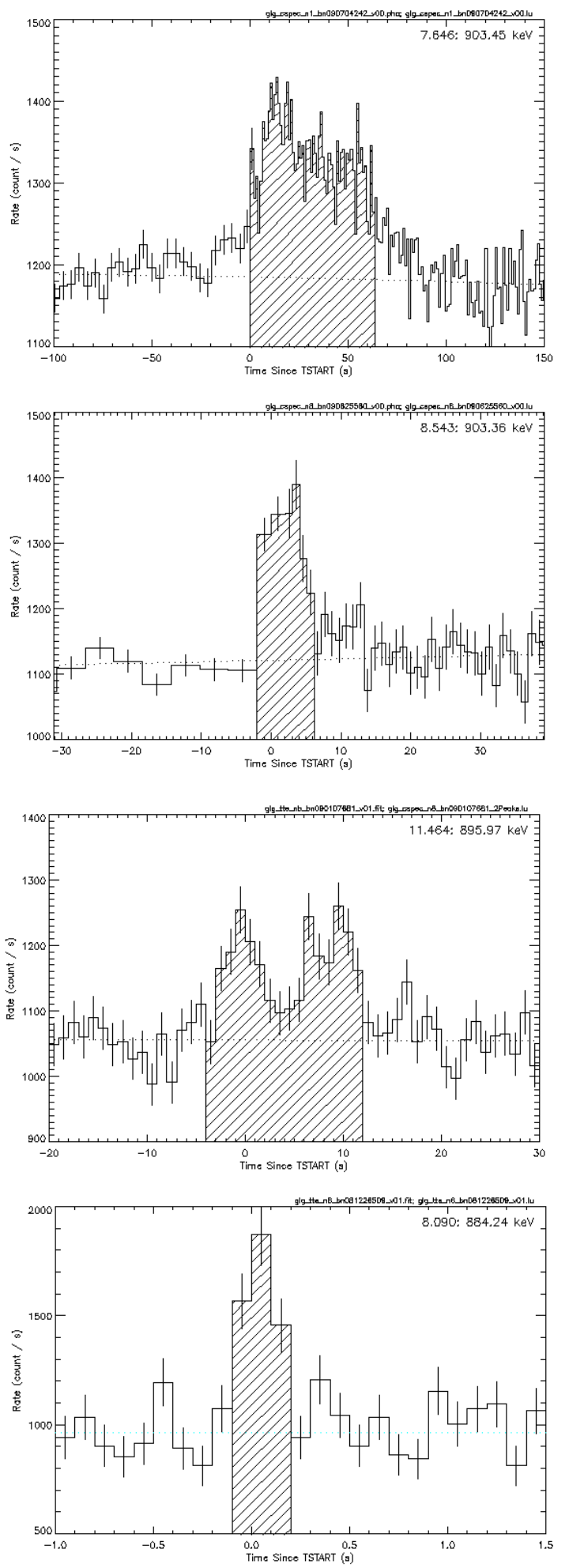

Figure 1. Lightcurves from ISGRI (left) and GBM (right); (a,b) GRB090704; (c,d) GRB090625B; (e,f) GRB090107B; (g,h) GRB081226B 


\section{Results}

The joint instrument fit results are presented in Table 2 and Figure 2.

\begin{tabular}{|c|c|c|c|c|c|c|c|c|}
\hline Burst & Instrument & Model & Fit & PL Index & HE CutOff & Norm & $\chi^{2} /$ d.o.f. & Constant \\
\hline GRB081226B & ISGRI & PL & Joint & $1.50_{-0.11}^{+0.13}$ & - & $5.67_{-4.41}^{+4.30}$ & $593 / 586$ & $0.76_{-0.19}^{+0.28}$ \\
\hline GRB090107B & ISGRI & CutOff PL & Joint & 1.27 & $187.66_{-6661}^{+152.43}$ & $1.94_{-0}^{+1}$ & $544 / 465$ & $1.07_{-0.11}^{+0.13}$ \\
\hline GRB090625B & ISGRI & CutOff PL & Joint & $0.69_{-0.29}^{+0.18}$ & $92.26_{-27.20}^{+22.60}$ & $0.42_{-0.42}^{+0.31}$ & $466 / 465$ & $1.11_{-0.11}^{+0.11}$ \\
\hline GRB090704 & ISGRI & CutOff PL & Joint & $1.16_{-0.10}^{+0.09}$ & $248.16_{-62.35}^{+92.37}$ & $1.05_{-0.29}^{+0.35}$ & $597 / 465$ & $1.08_{-0.07}^{+0.07}$ \\
\hline
\end{tabular}

Table 2. Best fit results from modelling the burst spectra with a simple PL and a COPL. 90\% uncertainties are shown for results.

The normalisation constant between ISGRI and GBM, with GBM being set to 1 , varies by $\sim$ 10\%. This shows a broad agreement between the two instruments. GRB081226B does not fit the general trend of the data and this may be due to poor statistics which can be inferred from the large uncertainty on the normalisation constant.

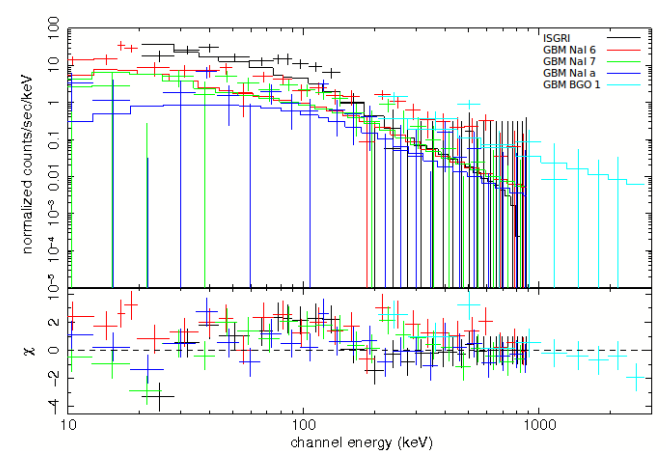

(a)

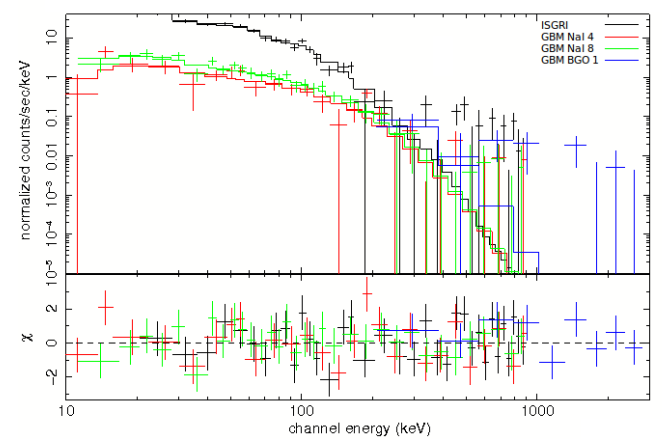

(c)

Figure 2. Best fit combined spectra obtained from ISGRI and GBM; (a)GRB081226B - Simple PL; (b)GRB090107B - COPL; (c)GRB090625B - COPL; (d)GRB090704 COPL.The data were binned for illustrative purposes by combining bins to a 3 sigma significance or by combining 5 consecutive bins, whichever condition was satisfied first.

Fermi was proposed to have a mission lifetime of 5 years starting in 2008 and finishing in 2013, with a goal of 10 years of operations. INTEGRAL has its mission lifetime extended until the end of 2014, which, given the mean rate of common GRBs at $1 \sim$ every 4 months, provides sufficient mission overlap time for more simultaneously observable bursts to occur. These GRBs can be added to the sample of bursts and by using a larger sample the objectives of this study can be achieved in greater detail. 


\section{Acknowledgments}

Dave Tierney acknowledges support from Science Foundation Ireland under grant number 09-RFPAST-2400. Suzanne Foley acknowledges the support of the Irish Research Council for Science, Engineering and Technology, cofunded by Marie Curie Actions under FP7.

\section{References}

[1] C. Winkler et al. 2003, The INTEGRAL mission, Astronomy and Astrophysics, 411, L1-L6

[2] P. Ubertini et al. 2003, IBIS: The Imager on-board INTEGRAL, Astronomy and Astrophysics, 411, L131-L139

[3] http://www.isdc.unige.ch/integral/download/osa_doc

[4] C. Meegan et al. 2009, The Fermi Gamma-ray Burst Monitor, The Astrophysical Journal, 702, 791-804

[5] E. Bissaldi et al. 2009, Experimental Astronomy, 24, 47-88

[6] A. Von Kienlin et al. 2009, Using GRB 080723B to cross-calibrate Fermi/GBM and INTEGRAL, American Institute of Physics Conference Series, 1133, 446-448

[7] M. Stamatikos et al. 2009, The Cross-Calibration of Swift-BAT and Fermi-GBM via Correlative Spectral Analysis of GRBs, ArXiv e-prints

[8] N. Gehrels et al. 2004, The Swift Gamma-Ray Burst Mission, The Astrophysical Journal, 611, 1005-1020

[9] G. Vedrenne et al. 2003, SPI: The spectrometer aboard INTEGRAL, Astronomy and Astrophysics, 411, L63-L70

[10] B. Atwood et al. 2009, The Large Area Telescope on the Fermi Gamma-Ray Space Telescope Mission, The Astrophysical Journal, 697, 1071-1102

[11] S. Mereghetti et al. 2008, GRB Coordinates Network 8734

[12] P. Evans \& E. Hoversten 2008, GRB Coordinates Network 8740

[13] E. Bissaldi \& S. McBreen 2008, GRB Coordinates Network 8751

[14] D. Gotz et al. 2009, GRB Coordinates Network 8786

[15] G. Stratta et al. 2009, GRB Coordinates Network 8791

[16] A. Goldstein et al. 2009, GRB Coordinates Network 8793

[17] Y. Nakagawa et al. 2009, GRB Coordinates Network 8794

[18] D. Gotz et al. 2009, GRB Coordinates Network 9572

[19] J. Mao et al. 2009, GRB Coordinates Network 9580

[20] A. Rau 2009, GRB Coordinates Network 9583

[21] S. Mereghetti et al. 2009, GRB Coordinates Network 9620

[22] S. McBreen 2009, GRB Coordinates Network 9627

[23] http://fermi.gsfc.nasa.gov/ssc/data/analysis/user/

[24] K. Araund et al. 1996, XSPEC: The First Ten Years, Astronomical Data Analysis Software and Systems $V, 101,17$

[25] D. Band et al. 1993, BATSE observations of gamma-ray burst spectra. I - Spectral diversity, The Astrophysical Journal, 413, 281-292 